\title{
Long-term oncologic outcomes of papillary thyroid microcarcinoma according to the presence of clinically apparent lymph node metastasis: a large retrospective analysis of 5,348 patients
}

This article was published in the following Dove Press journal: Cancer Management and Research

\section{Jung Bum Choi, ${ }^{1, *}$ Woo} Kyung Lee, ${ }^{2,3, *}$ Seul Gi Lee, ${ }^{2,4}$ Haengrang Ryu, ${ }^{5}$ Cho Rok Lee, ${ }^{4}$ Sang Wook Kang, ${ }^{4}$ Jong Ju Jeong, ${ }^{4}$ Kee-Hyun Nam, ${ }^{4}$ Eun Jig Lee, ${ }^{2,3}$ Woong Youn Chung, ${ }^{4}$ Young Suk Jo, ${ }^{2,3}$ Jandee Lee ${ }^{4}$

'Department of Surgery, Pusan National University College of Medicine, Busan, South Korea; ${ }^{2}$ Brain Korea 21 PLUS Project for Medical Science, Yonsei University, Seoul, South Korea; ${ }^{3}$ Department of Internal Medicine, Severance Hospital, Yonsei Cancer Center, Yonsei University College of Medicine, Seoul, South Korea; ${ }^{4}$ Department of Surgery, Open NBI Convergence Technology Research Laboratory, Yonsei Cancer Center, Severance Hospital, Yonsei University College of Medicine, Seoul, South Korea; ${ }^{5}$ Department of Surgery, Hongik Hospital, Seoul, South Korea

*These authors contributed equally to this work

Correspondence: Young Suk Jo Department of Internal Medicine, Yonsei Cancer Center, Severance Hospital, Yonsei University College of Medicine, 120-752 Seoul, South Korea

Tel +82 222280888

Fax +8223936884

Email joys@yuhs.ac

Jandee Lee

Department of Surgery, Open NBI Convergence Technology Research Laboratory, Yonsei Cancer Center, Yonsei University

College of Medicine, 03722 Seoul, South Korea Tel +82 222282100

$\mathrm{Fax}+8223138289$

Email jandee@yuhs.ac
Purpose: Active surveillance (AS) of low-risk papillary thyroid microcarcinoma (PTMC) may reduce the risk of overtreatment of clinically insignificant cancer. However, the absence of predictor for the progression of PTMC resulted in treatment delay and potentially compromising cure of aggressive disease. Therefore, to anticipate potential damage of delayed surgery, we investigated the oncologic outcomes of patients with low-risk PTMC initially eligible for AS except clinically apparent lymph node metastasis (LNM), imitating delayed surgery with neck dissection.

Materials and methods: A total of 5,348 patients, enrolled between 1987 and 2016, with low-risk PTMC initially eligible for AS were included regardless of LNM. We classified our study patients into two groups: Group I, lobectomy with prophylactic central cervical node dissection; Group II, total thyroidectomy with modified radical neck dissection for LNM. In addition, we investigated the oncological outcomes of patients with second-wave surgery due to lateral lymph node recurrence (Group III, subgroup of Group I).

Results: Group I showed more favorable clinicopathological characteristics compared with Group II. In Group I, only 29 (0.58\%) of 4,927 patients underwent second-wave surgery with neck dissection for lateral lymph node recurrences, whereas in Group II, all 22 (5.23\%) of 421 patients underwent second-wave selective node dissection because of nodal recurrence. Diseasefree survival rates were significantly different between Groups I and II $(P<0.05)$. Of note, the recurrence rate of Group II was still significantly higher than that of Group III $(5.2 \%$ vs $0 \%$, respectively; $P=0.021$ ). In addition, Kaplan-Mayer survival analysis indicated poor disease-free survival rates in Group II compared with Group III $(P<0.05)$.

Conclusion: The long-term treatment outcome of PTMC without LNM was favorable even if the recurrence occurs during follow-up period compared with that of PTMC with LNM. It should be noted that AS might be able to cause poor prognosis due to clinically apparent LNM. Keywords: active surveillance, papillary thyroid microcarcinoma, lymphatic metastasis, neoplasm recurrence

\section{Introduction}

Recent studies have suggested that active surveillance (AS) for carefully selected patients with low-risk localized papillary thyroid carcinoma (PTC), especially papillary thyroid microcarcinoma $(\mathrm{PTMC}) \leq 1 \mathrm{~cm}$ in maximum diameter, may reduce the 
risk of overtreatment of clinically insignificant cancer. ${ }^{1,2}$ Because the majority of these small thyroid cancers progress slowly or not at all, it is crucial to reassess the conventional management approach of immediate surgery for all PTCs. ${ }^{1,3,4}$ Accordingly, the American Thyroid Association (ATA) stated that AS should be considered as an initial approach instead of definitive intervention in patients with low-risk PTMC, those at high operative risk because of comorbidity, and those with a short life expectancy. ${ }^{5}$

Although optimal criteria to define AS eligibility have not been agreed upon, most AS protocols rely primarily on initial tumor size, tumor extension, metastases, patient's perspective, and even on the presence of supportive healthcare members. These candidates should be closely monitored via serial highresolution neck ultrasonography (US), physical examination, and/or other imaging studies during the follow-up while maintaining the option of definitive therapy for patients who are reclassified as patients with higher risk. ${ }^{6}$

However, in 2\%-15\% of patients deemed eligible for AS, tumor characteristics or extent may be underestimated, resulting in treatment delay and potentially compromising cure for patients with aggressive disease. ${ }^{7-13}$ However, no definite biological or clinical parameters currently exist to distinguish low-risk indolent PTMC from potentially aggressive PTMC. Therefore, some patients initially eligible for AS could present disease progression such as lymph node metastasis (LNM), considering that is not possible to predict aggressive disease and $\mathrm{AS}$ is inadequate for these patients.

Because AS of low-risk PTMC has been validated in limited instances over short periods, there is currently little research on long-term oncologic outcomes. The present study compared the incidences of recurrence in patients with lowrisk PTMC initially eligible for AS regardless of LNM. By this approach, we could investigate the oncologic outcome of imitate delayed surgery after the detection of clinically apparent LNM during AS. To achieve our goal, we have assessed the oncologic outcomes of a large retrospective cohort of 5,348 patients with low-risk PTMC with a followup of up to 25 years.

\section{Materials and methods Study cohort}

Between 1987 and 2016, a total of 18,404 consecutive patients diagnosed with PTMC underwent surgical treatment at our hospital. We reviewed the medical records of these patients using the endocrine surgery database at Yonsei University College of Medicine. This study was approved by the Institutional Review Board, and the requirements for patient approval and informed consent were waived due to retrospective design of the study. The anonymous data were kept confidential, and only researchers of this study had a private access to the data. First, we excluded $10,349(56.2 \%)$ patients who underwent total thyroidectomy (TT) and central compartment neck node dissection (CCND) with preoperative high-risk PTMC showing one or more aggressive clinical features such as neck irradiation and family history of thyroid cancer. In addition, we excluded the patients who underwent TT with CCND due to therapeutic strategy such as postoperative radioactive iodine therapy based on the final pathologic stage (Figure 1). Of the remaining 8,055 patients, we identified 7,264 (39.4\%) who underwent lobectomy with prophylactic CCND, our standard procedure for low-risk PTMC. Of them, 4,927 (26.8\%) patients (Group I) met the criteria for AS suggested by the thyroid cancer management team at Memorial Sloan Kettering Cancer Center. ${ }^{6}$ Among them, 781 (15.9\%) were defined as ideal candidates and 4,146 (84.1\%) as appropriate candidates (Figure 1). We have also enrolled patients who met the criteria for consideration as a candidate for AS except clinically apparent LNM. In our study cohort, a total of 808 (4.4\%) patients with PTMC underwent TT and modified radical neck dissection (MRND) due to lateral neck node metastasis. Of these, we excluded 387 (2.1\%) patients with preoperatively detected extrathyroidal extension (ETE), T4 tumors, clinical apparent central LNM, and distant metastasis as inappropriate candidates for AS. Finally, the remaining 421 patients (Group II) met the AS criteria except clinically apparent LNM: 52 (12.4\%) were ideal candidates, and 369 (87.6\%) were appropriate candidates. Therefore, we postulated our patients divided into two groups imitating initial definite intervention (Group I) and delayed intervention after unfavorable nodal metastasis (Group II) (Figure 1). Postoperative pathological evaluation did not show any case with positive surgical margin.

\section{Postoperative management and follow-up}

After surgery, the patients who underwent TT with MRND underwent radioactive iodine ablation (100-200 $\mathrm{mCi}$ ). We have regularly followed all patients by US and serum thyroglobulin testing at 6- or 12-month intervals to check for local recurrences. Each patient also had an annual chest X-ray or a computed tomography scan to detect potential lung metastases. Structural recurrent disease was defined as pathologically or cytologically proven recurrent or metastatic lesions and/or the appearance of metastatic lesions in other distant organs on imaging studies. In this study, because 


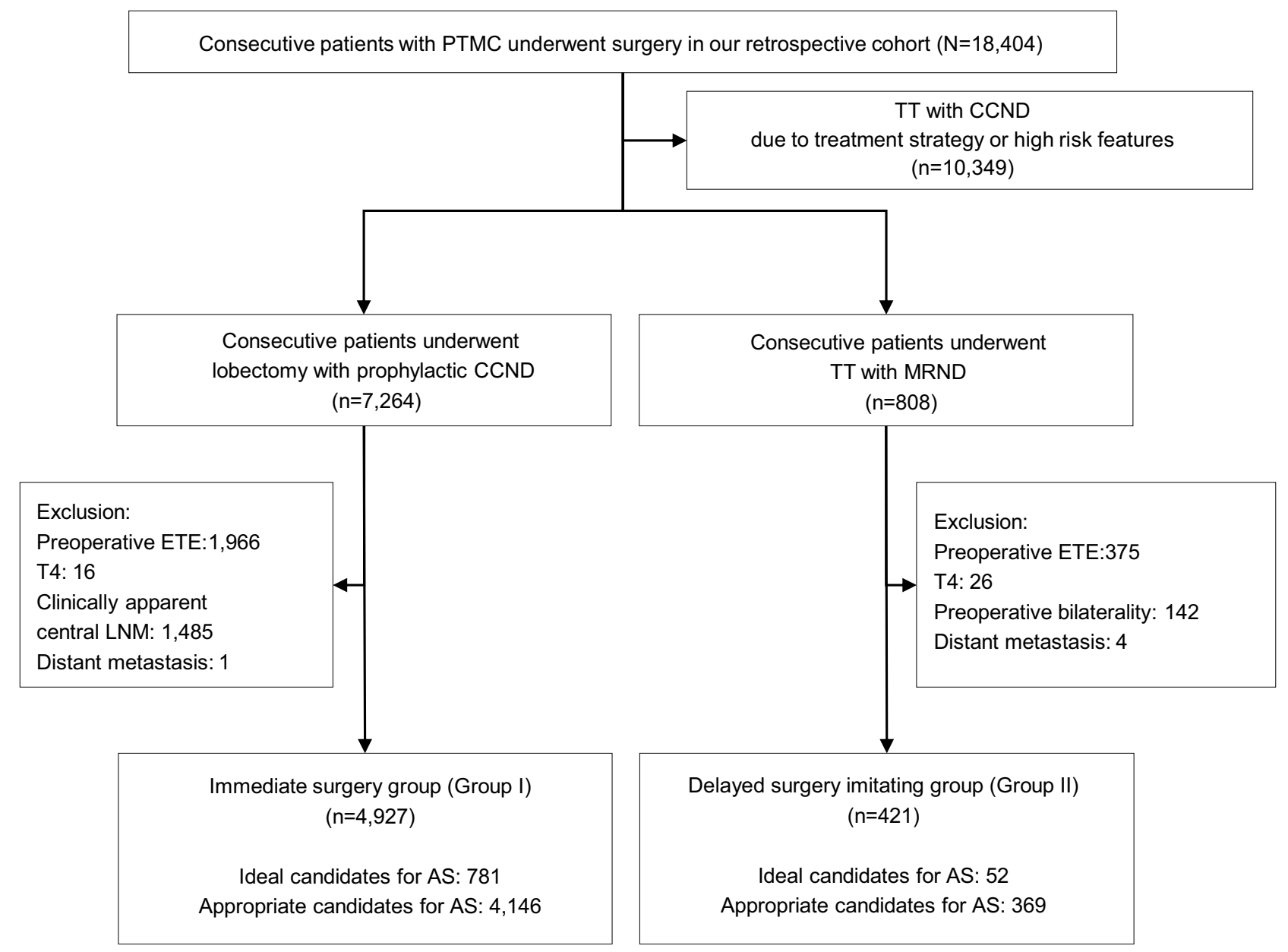

Figure I Scheme of defining group indicating immediate surgery group of ideal or appropriate candidates for AS and delayed surgery imitating group of ideal or appropriate candidates for AS except accompanying with clinically apparent LNM.

Abbreviations: AS, active surveillance; CCND, central compartment neck node dissection; ETE, extrathyroidal extension; LNM, lymph node metastasis; MRND, modified radical neck dissection; PTMC, papillary thyroid microcarcinoma; TT, total thyroidectomy.

most of patients underwent lobectomy initially, we did not consider recurrence if structural disease was not identified. Disease-free survival (DFS) was defined as the time interval from initial surgery to the detection of structural recurrent disease.

\section{Statistical analysis}

Data were expressed as mean \pm SD or $\mathrm{n}(\%)$ for descriptive statistics. A $t$-test was used for continuous variables. Pearson's chi-squared test and Fisher's exact test were used for categorical variables, appropriately. Kaplan-Meier survival analysis was used to estimate DFS. Statistical significance was set at $P<0.05$. Statistical analyses were performed using IBM SPSS statistics version 23.0 (SPSS Inc., Chicago, IL, USA), and R version 3.1.3 (http://www.R-project.org).

\section{Results Oncologic outcomes of study cohort} In Group I, 4,927 patients with low-risk PTMC, potential candidates for AS, underwent immediate lobectomy with prophylactic CCND (Figure 2). During follow-up period, $48(0.97 \%)$ patients of Group I showed structural recurrent disease. Nineteen $(0.38 \%)$ patients underwent second-wave completion thyroidectomy with CCND and 29 (0.59\%) patients underwent second-wave completion thyroidectomy with MRND, according to the level of LNM. In the case of Group II, 421 patients underwent TT with MRND (Group II). Twenty-two (5.2\%) patients of Group II presented neck node recurrence followed by second-wave selective node dissection. The median follow-up period was 163 months (range, 121-300 months). 


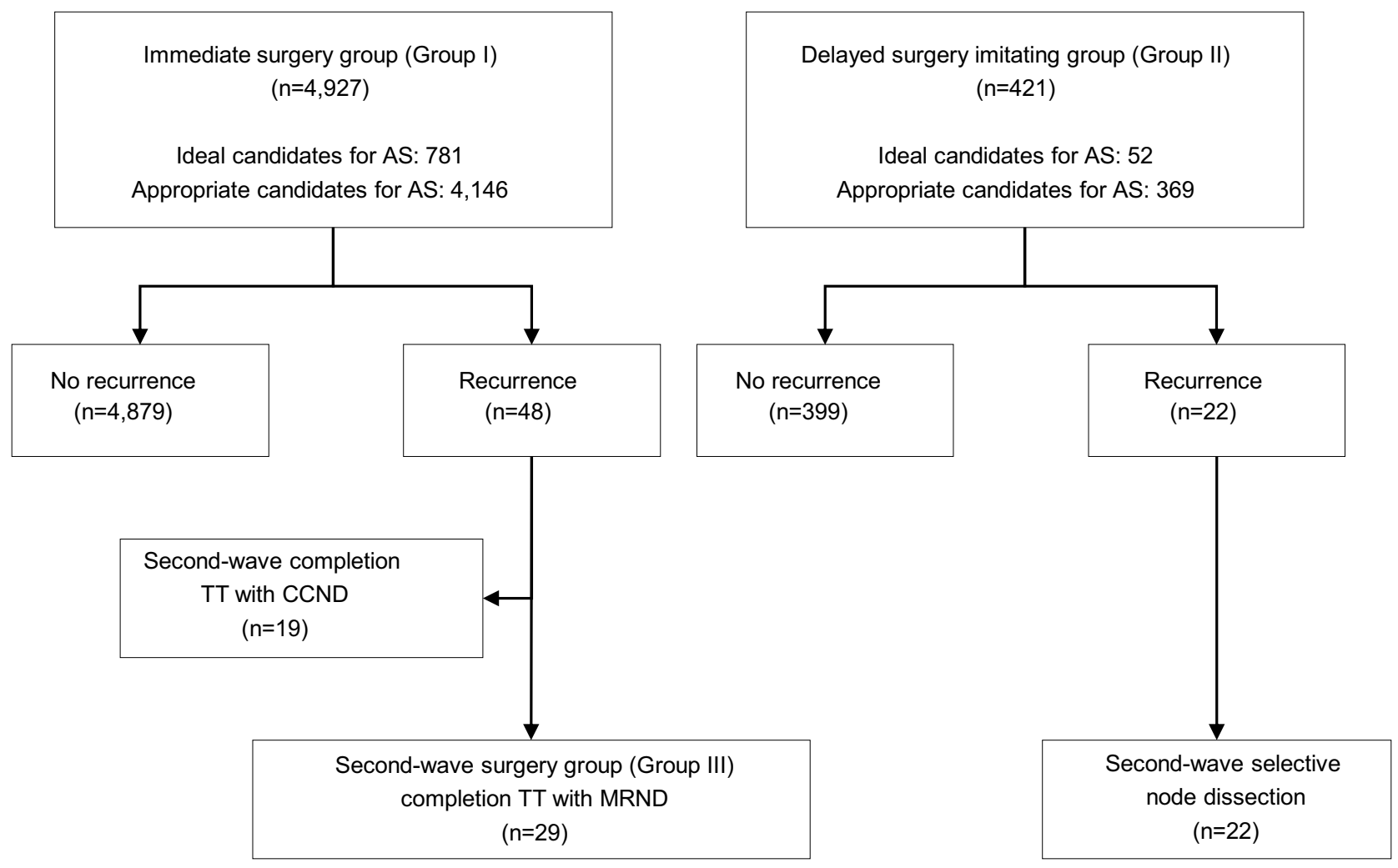

Figure 2 Flow chart presenting the oncologic outcomes of immediate surgery group and delayed surgery imitating group with low-risk PTMC potentially eligible for AS. Abbreviations: AS, active surveillance; CCND, central compartment neck node dissection; MRND, modified radical neck dissection; PTMC, papillary thyroid microcarcinoma; TT, total thyroidectomy.

\section{Comparisons of clinicopathological characteristics between Group I and Group II}

In addition to recurrence, the presence or absence of LNM produced remarkable clinical differences. Compared with Group I, Group II presented old age (46.1 \pm 8.7 vs $47.5 \pm 1.7$ years, respectively; $P=0.001)$ and frequent male gender (18.2\% vs $27.7 \%$, respectively; $P<0.001)$. Mean primary tumor size of Group II was larger than that of Group I $(0.7 \pm 0.2$ vs $0.5 \pm 0.2 \mathrm{~cm}$, respectively; $P<0.001)$. Multifocality (46.7\% vs $10.1 \%$, respectively; $P<0.001$ ) and microscopic ETE (57.7\% vs $26.8 \%$, respectively; $P<0.001)$ were also frequently observed in Group II than in Group I. We also found obvious differences in other pathological staging characteristics, including T-stage $(P<0.001)$, N-stage $(P<0.001)$, and tumor node metastasis (TNM) staging $(P<0.001)$ between Groups I and II. Evaluation of lymph node ratio (LNR), defined as the number of metastatic lymph nodes (LNs) divided by the total number of LNs retrieved from the central compartment (central LNR) and with nodes from lateral compartments (total LNR), showed that Group II tended to have a higher central and total LNR than Group I (Table 1). During follow-up period as shown in Figure 2, structural recurrent disease was detected in $48(0.9 \%)$ cases out of Group I and $22(5.2 \%)$ cases out of Group II $(P<0.001)$. Two patients of Group II died due to disease progression of distant metastasis; however, there was no disease-specific mortality in Group I $(P=0.006)$. Using the Kaplan-Meier method, we found that 20-year DFS rates were significantly different between Groups I and II $(P<0.001$, Figure 3A).

\section{Impacts of second-wave completion thyroidectomy with MRND on clinicopathological characteristics}

We also evaluated the clinicopathological characteristics and oncologic outcomes of patients who underwent second-wave MRND due to lateral LN recurrence following initial immediate intervention $(n=29)$ (Group III, a subgroup of Group I). There were still obvious differences between Group III and Group II in primary tumor size $(0.6 \pm 0.3$ vs $0.7 \pm 0.2 \mathrm{~cm}$, respectively; $P=0.001$ ) and TNM staging (more frequent Stage IV in Group II, $P<0.001$ ), but other clinicopathological 
Table I Comparison of clinicopathological characteristics between patients with low-risk PTMC without LNM who underwent lobectomy with prophylactic CCND ( $N=4,927)$ (Group I) and patients with low-risk PTMC except clinically apparent LNM who underwent total thyroidectomy with MRND ( $N=42 I)$ (Group II)

\begin{tabular}{|c|c|c|c|}
\hline & Group I & Group II & $P$-value \\
\hline Age (years), mean $\pm S D$ & $46.1 \pm 8.7$ & $47.5 \pm 1.7$ & 0.001 \\
\hline Male/female ratio & $90 \mathrm{I} / 4,026$ (I8.2\%/8I.8\%) & II $17 / 304$ (27.7\%/72.3\%) & $<0.001$ \\
\hline Tumor size $(\mathrm{cm})$, mean $\pm S D$ & $0.5 \pm 0.2$ & $0.7 \pm 0.2$ & 0.001 \\
\hline Multifocality & $496(10.1 \%)$ & 197 (46.7\%) & $<0.001$ \\
\hline Microscopic ETE & I,326 (26.8\%) & $243(57.7 \%)$ & $<0.001$ \\
\hline Central node metastasis & $\mathrm{I}, 024$ (20.7\%) & $308(73.1 \%)$ & $<0.001$ \\
\hline Retrieval LN & $4.8 \pm 3.7$ & $8.5 \pm 6.6$ & $<0.001$ \\
\hline Central LNR & $0.11 \pm 0.03$ & $0.53 \pm 0.22$ & 0.013 \\
\hline Lateral node metastasis & - & $421(100 \%)$ & - \\
\hline Retrieval LN & - & $30.1 \pm 13.8$ & - \\
\hline LNR & - & $0.23 \pm 0.15$ & - \\
\hline Total LNR & $0.1 I \pm 0.03$ & $0.30 \pm 0.12$ & 0.031 \\
\hline Pathologic type & & & 0.017 \\
\hline Papillary & 4,927 (100\%) & 421 (100\%) & \\
\hline Classic & $4,752(96.5 \%)$ & $40 I(95.2 \%)$ & \\
\hline Follicular variant & $165(3.3 \%)$ & $17(4.1 \%)$ & \\
\hline Diffuse sclerosing & $10(0.2 \%)$ & $3(0.7 \%)$ & \\
\hline T stage & & & $<0.001$ \\
\hline TI & $3,602(73.1 \%)$ & 179 (70.3\%) & \\
\hline $\mathrm{T} 2$ & $2(0.02 \%)$ & 0 & \\
\hline T3 & I,323 (26.7\%) & $242(37 \%)$ & \\
\hline $\mathrm{T} 4$ & 0 & 0 & \\
\hline$N$ stage & & & $<0.001$ \\
\hline No & 3,903 (79.2\%) & 0 & \\
\hline $\mathrm{Nla}$ & $\mathrm{I}, 024(20.7 \%)$ & 0 & \\
\hline NIb & 0 & $421(100 \%)$ & \\
\hline TNM staging & & & $<0.001$ \\
\hline Stage I & $4, \mathrm{I} \mid 4(83.5 \%)$ & $226(53.6 \%)$ & \\
\hline Stage II & $3(0.001 \%)$ & 0 & \\
\hline Stage III & $810(16.4 \%)$ & $12(2.8 \%)$ & \\
\hline Stage IV & 0 & $183(43.4 \%)$ & \\
\hline Distant metastasis & 0 & $2(0.4 \%)$ & 0.006 \\
\hline Recurrence & 48 (0.9\%) & $22(5.2 \%)$ & $<0.001$ \\
\hline Disease-specific mortality rate & 0 & $2(0.4 \%)$ & 0.006 \\
\hline
\end{tabular}

Abbreviations: CCND, central compartment neck node dissection; ETE, extrathyroidal extension; LN, lymph node; LNM, lymph node metastasis; LNR, lymph node ratio; MRND, modified radical neck dissection; PTMC, papillary thyroid microcarcinoma; TNM, tumor node metastasis.

findings showed no difference. During follow-up period, no recurrence after second-wave surgery and no fatal case were observed in Group III (Table 2). The DFS curve also showed that 20-year DFS rates were significantly different between Groups III and II ( $P=0.031$, Figure 3B).

\section{Discussion}

Given the high prevalence of low-risk PTMC in the general population and its relative indolent nature, AS by specialized medical teams can be an evolving option in selected patients. ${ }^{414}$ Using the AS strategy developed in Kuma Hospital in Japan, patients with low-risk PTMC with high-risk features such as locoregional invasion, distant metastasis, and signs of aggressive disease on high-quality US were excluded. ${ }^{2-4,15,16}$ Recently, ATA guidelines also introduced AS as an alternative strategy to immediate thyroid surgery in patients with very low-risk tumors, and patients are classified as ideal, appropriate, or inappropriate candidates for AS. ${ }^{5,6}$ To assess patients eligible for AS and medical team characteristics, initial and follow-up evaluations require serial neck US to evaluate the size and location of the thyroid cancer and the status of the cervical LNs. ${ }^{5,6}$ However, in 2\%-15\% of patients initially eligible for AS, imaging studies and fineneedle aspiration cytology can underestimate tumor characteristics or extent, resulting in treatment delay and potentially compromising cure for patients with aggressive disease. ${ }^{7-13}$ 


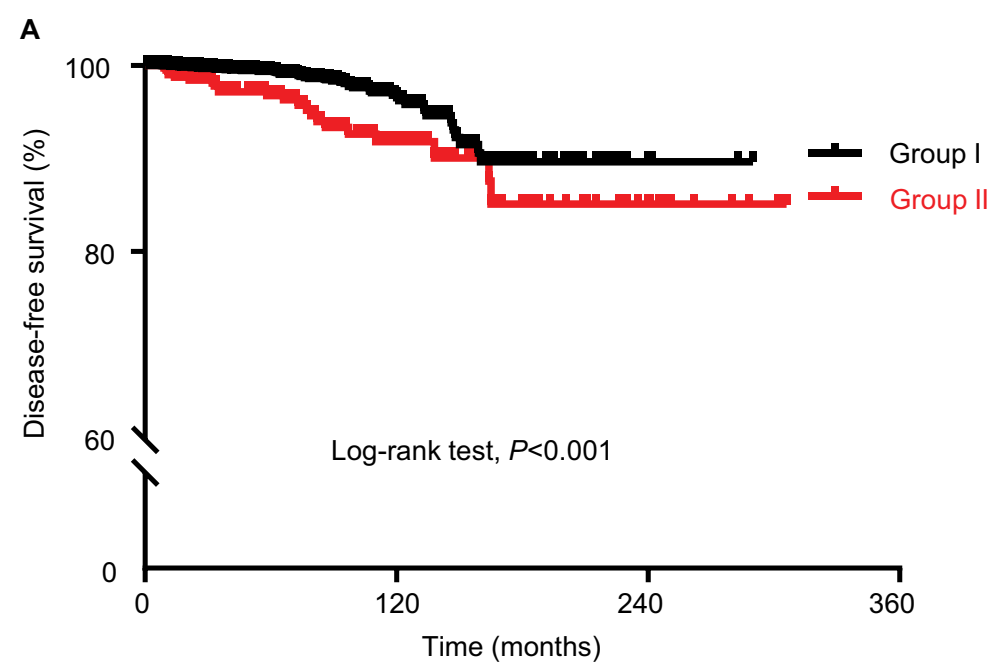

B

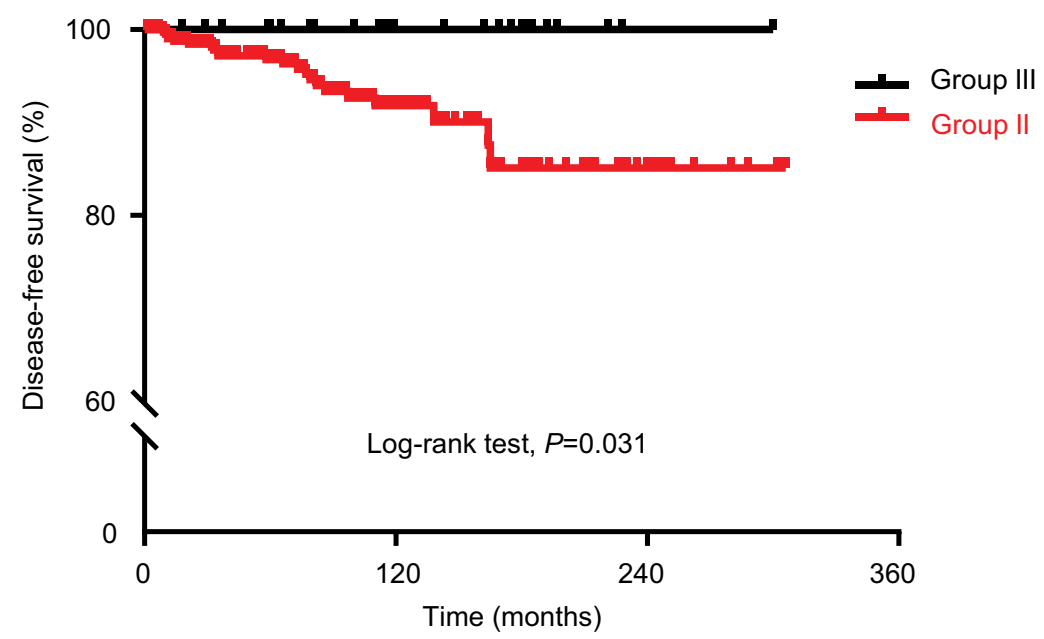

Figure 3 Comparison of disease-free survival according to patients' Group.

Notes: (A) Comparison of disease-free survival of patients with low-risk PTMC who underwent lobectomy with prophylactic central cervical node dissection (Group I) with that of patients with low-risk PTMC except clinically apparent lymph node metastasis who underwent total thyroidectomy with modified radical neck dissection (Group II). (B) Comparison of disease-free survival of patients with second-wave surgery due to lateral LN recurrence (Group III, subgroup of Group I) with that of Group II. P-value was determined by the log-rank test.

Abbreviation: PTMC, papillary thyroid microcarcinoma.

In fact, AS strategy in low-risk PTMC has been suggested by a Japanese group based on single observational studies, and concerns remain about disease progression during AS, especially in patients with an aggressive phenotype. ${ }^{17-19}$ Although AS has existed for approximately 10 years, it was only recently added to the international guidelines as a viable management strategy. ${ }^{5,14}$ One of the reasons for the delay was the persisting lack of long-term follow-up data from
AS cohorts. Additionally, no comparative analysis has been performed to evaluate the long-term oncologic outcomes in patients with low-risk PTMC according to the timing of the surgery; immediate surgical intervention vs delayed surgery following clinically apparent LNM.

In this study, we aimed to generate imitating group of AS who underwent delayed surgery after detection of clinically apparent LNM with retrospective-based setting. In addition, 
Table 2 Comparison of clinicopathological characteristics in patients with low-risk PTMC who underwent second-wave MRND following initial immediate surgery $(\mathrm{N}=29)$ (Group III) and patients with low-risk PTMC except clinically apparent LNM who underwent total thyroidectomy with MRND ( $\mathrm{N}=42 \mathrm{I})$ (Group II)

\begin{tabular}{|c|c|c|c|}
\hline & Group II & Group III & $P$-value \\
\hline Age (years), mean $\pm S D$ & $47.5 \pm 1.7$ & $47 \pm 9.7$ & 0.652 \\
\hline Male/female ratio & II $7 / 304$ (27.7\%/72.3\%) & $7 / 22(24.1 \% / 75.9 \%)$ & 0.526 \\
\hline Tumor size $(\mathrm{cm})$, mean $\pm S D$ & $0.7 \pm 0.2$ & $0.6 \pm 0.3$ & 0.001 \\
\hline Multifocality & 197 (46.7\%) & 18 (62\%) & 0.316 \\
\hline Microscopic ETE & $243(57.7 \%)$ & $10(34.4 \%)$ & 0.067 \\
\hline Central node metastasis & $308(73.1 \%)$ & $23(79.3 \%)$ & 0.663 \\
\hline Retrieval LN & $8.2 \pm 6.6$ & $8.2 \pm 1.9$ & 0.876 \\
\hline Central LNR & $0.53 \pm 0.22$ & $0.5 I \pm 0.32$ & 0.352 \\
\hline Lateral node metastasis & $421(100 \%)$ & $29(100 \%)$ & 1.0 \\
\hline Retrieval LN & $30.1 \pm 13.8$ & $26.7 \pm 16.1$ & 0.031 \\
\hline LN ratio & $0.23 \pm 0.15$ & $0.24 \pm 0.35$ & 0.913 \\
\hline Total LNR & $0.30 \pm 0.12$ & $0.30 \pm 0.18$ & 0.986 \\
\hline Pathologic type & & & 0.592 \\
\hline Papillary & $421(100 \%)$ & $29(100 \%)$ & \\
\hline Classic & $40 \mathrm{I}(95.2 \%)$ & $27(93.1 \%)$ & \\
\hline Follicular variant & $17(4.1 \%)$ & $2(6.8 \%)$ & \\
\hline Diffuse sclerosing & $3(0.7 \%)$ & 0 & \\
\hline T stage & & & 1.0 \\
\hline TI & 179 (70.3\%) & 19 (70.3\%) & \\
\hline $\mathrm{T} 2$ & 0 & 0 & \\
\hline T3 & $242(37 \%)$ & $10(37 \%)$ & \\
\hline $\mathrm{T} 4$ & 0 & 0 & \\
\hline$N$ stage & & & 1.0 \\
\hline No & 0 & 0 & \\
\hline $\mathrm{Nla}$ & 0 & 0 & \\
\hline NIb & $421(100 \%)$ & $29(100 \%)$ & \\
\hline TNM staging & & & $<0.001$ \\
\hline Stage I & $226(53.6 \%)$ & 22 (75.9\%) & \\
\hline Stage II & 0 & 0 & \\
\hline Stage III & $12(2.8 \%)$ & $6(22.2 \%)$ & \\
\hline Stage IV & $183(43.4 \%)$ & I (3.7\%) & \\
\hline Distant metastasis & $2(0.4 \%)$ & I (3.7\%) & 0.182 \\
\hline Recurrence & $22(5.2 \%)$ & 0 & 0.021 \\
\hline Disease-specific mortality rate & $2(0.4 \%)$ & 0 & $\mathrm{I} .0$ \\
\hline
\end{tabular}

Abbreviations: ETE, extrathyroidal extension; LN, lymph node; LNM, lymph node metastasis; LNR, lymph node ratio; MRND, modified radical neck dissection; PTMC, papillary thyroid microcarcinoma; TNM, tumor node metastasis.

to understand the risk of delayed operation, we compared this imitating group (Group II) with immediate surgery group (Group I). Furthermore, we analyzed the oncologic outcome of second-wave surgery group because of local recurrence (Group III). Although this study has limitations because of its retrospective nature, to the best of our knowledge, our study provides the first evidence of large population-based long-term oncologic outcomes in 5,348 patients with low-risk PTMC who were initially eligible for AS.

In the comparison of baseline characteristics, we found remarkable difference between two groups (Group I vs Group II). In fact, according to the study design, Group I can be regarded as an immediate operated group without AS. And, Group II can be considered as delayed surgery group. The immediate intervention group presented striking favorable characteristics compared with delayed surgical intervention group. Of note, recurrence and even disease-specific mortality were different between two groups. All these data consistently represent the risk of delayed surgery after the development of clinically apparent LNM.

The ability to identify patients with indolent PTMC without progressive disease earlier and more accurately is likely to result in improved oncologic outcome such as lower metastasis and lower disease recurrence. It would also offer reassurance to patients without higher-risk disease when determining AS candidates. Both improved imaging technology and molecular biomarkers show promise in this regard. ${ }^{7,20,21}$ Nevertheless, thus far, no definite biological or 
clinical parameters are available that can distinguish low-risk from potentially aggressive thyroid cancer, and the long-term oncological effect of delayed initial intervention has not been evaluated. For example, if follow-up imaging studies find cancer progression during AS, immediate intervention, including radical thyroid surgery with MRND, should be performed. However, the long-term oncological outcomes in patients with LNM who underwent primary-wave MRND following delayed initial intervention compared with secondwave MRND after initial immediate surgery have not been determined. In this study, the analysis of clinicopathological characteristics of second-wave surgery group (Group III) more strongly support our idea for the risk of delayed surgery. Although the patients of this group have to undergo repeated surgery, their oncologic outcome was more favorable than Group II. We believe that this difference is quite important because local recurrence presenting lateral LNM makes patients with same N1b stage but the oncologic outcome such as recurrence rate is still different. In fact, it may seem inappropriate to compare Groups II and III because of retrospective nature of this study. However, we have selected ideal or appropriate candidates for AS based on the preoperative characteristics of primary tumor, indicating that Group II is able to imitate disease progression group found by clinically apparent LNM. In this sense, although the comparison seems inappropriate, this comparison can reflect that there might be a risk of the delayed intervention group being found by LNM during AS.

This study might include some of patients with noninvasive follicular thyroid neoplasm with papillary-like nuclear features (NIFTP) by new WHO classification. However, the frequency of NIFTP was significantly lower in Asian series $(1.6 \%)$ compared with that of Western countries $(13.3 \%) .{ }^{22}$ To overcome this limitation, precise pathologic evaluation will be needed in the future studies.

\section{Conclusion}

Some low-risk PTMCs can progress significantly during the delayed intervention period after AS. We found that patients initially eligible for AS who underwent delayed surgical intervention had more aggressive disease and unfavorable oncologic outcomes than those who underwent initial intervention without AS. Considering the time point of MRND, patients who underwent primary-wave MRND following delayed initial intervention showed a significantly higher prevalence of recurrence and worse oncologic outcomes than those who underwent second-wave MRND after initial immediate surgery. Thus, additional prognostic factors to identify patients with an aggressive phenotype despite apparently low-risk PTMC are essential.

\section{Acknowledgments}

We thank Ji Young Kim and Hoyoung Kim for technical support. JL was supported by National Research Foundation of Korea (NRF) grants, funded by the Korean government (MEST) (NRF-2017R1D1A1A09000916) and by the Korean Thyroid Association Young Investigator Award 2016. YSJ was supported by National Research Foundation of Korea (NRF) grants, funded by the Korean government (MEST) (NRF-2015R1D1A1A01058912), by a grant from the Korea Health Technology R\&D Project through the Korea Health Industry Development Institute funded by the Ministry of Health \& Welfare, Republic of Korea (HI15C2334), and by the "Donghwa Holdings" Faculty Research Assistant Program of Yonsei University College of Medicine for 2016 (6-2016-0160).

\section{Disclosure}

The authors report no conflicts of interest in this work.

\section{References}

1. Sugitani I, Toda K, Yamada K, Yamamoto N, Ikenaga M, Fujimoto Y. Three distinctly different kinds of papillary thyroid microcarcinoma should be recognized: our treatment strategies and outcomes. World $J$ Surg. 2010;34(6):1222-1231.

2. Ito $\mathrm{Y}$, Miyauchi A, Inoue $\mathrm{H}$, et al. An observational trial for papillary thyroid microcarcinoma in Japanese patients. World J Surg. 2010;34(1): $28-35$.

3. Ito Y, Miyauchi A, Kihara M, Higashiyama T, Kobayashi K, Miya A. Patient age is significantly related to the progression of papillary microcarcinoma of the thyroid under observation. Thyroid. 2014;24(1): $27-34$.

4. Ito Y, Uruno T, Nakano K, et al. An observation trial without surgical treatment in patients with papillary microcarcinoma of the thyroid. Thyroid. 2003;13(4):381-387.

5. Haugen BR, Alexander EK, Bible KC, et al. 2015 American Thyroid Association Management Guidelines for Adult Patients with Thyroid Nodules and Differentiated Thyroid Cancer: The American Thyroid Association Guidelines Task Force on Thyroid Nodules and Differentiated Thyroid Cancer. Thyroid. 2016;26(1):1-133.

6. Brito JP, Ito Y, Miyauchi A, Tuttle RM. A clinical framework to facilitate risk stratification when considering an active surveillance alternative to immediate biopsy and surgery in papillary microcarcinoma. Thyroid. 2016;26(1):144-149.

7. Oda $\mathrm{H}$, Miyauchi A, Ito $\mathrm{Y}$, et al. Incidences of unfavorable events in the management of low-risk papillary microcarcinoma of the thyroid by active surveillance versus immediate surgery. Thyroid. 2016;26(1): 150-155.

8. Kwon H, Oh HS, Kim M, et al. Active surveillance for patients with papillary thyroid microcarcinoma: a single center's experience in Korea. J Clin Endocrinol Metab. 2017;102(6):1917-1925.

9. Miyauchi A. Clinical trials of active surveillance of papillary microcarcinoma of the thyroid. World J Surg. 2016;40(3):516-522.

10. Tuttle RM, Fagin JA, Minkowitz G, et al. Natural history and tumor volume kinetics of papillary thyroid cancers during active surveillance. JAMA Otolaryngol Head Neck Surg. 2017;143(10):1015-1020. 
11. Sugitani I, Fujimoto Y, Yamada K. Association between serum thyrotropin concentration and growth of asymptomatic papillary thyroid microcarcinoma. World J Surg. 2014;38(3):673-678.

12. Griffin A, Brito JP, Bahl M, Hoang JK. Applying criteria of active surveillance to low-risk papillary thyroid cancer over a decade: how many surgeries and complications can be avoided? Thyroid. 2017;27(4):518-523.

13. Kim HI, Jang HW, Ahn HS, et al. High serum TSH level is associated with progression of papillary thyroid microcarcinoma during active surveillance. J Clin Endocrinol Metab. 2017;103(2):446-451.

14. Takami H, Ito Y, Okamoto T, Onoda N, Noguchi H, Yoshida A. Revisiting the guidelines issued by the Japanese Society of Thyroid Surgeons and Japan Association of Endocrine Surgeons: a gradual move towards consensus between Japanese and western practice in the management of thyroid carcinoma. World J Surg. 2014;38(8):2002-2010.

15. Ito Y, Fukushima M, Kihara M, et al. Investigation of the prognosis of patients with papillary thyroid carcinoma by tumor size. Endocr $J$. 2012;59(6):457-464.
16. Ito $\mathrm{Y}$, Miyauchi A, Oda H. Low-risk papillary microcarcinoma of the thyroid: A review of active surveillance trials. Eur J Surg Oncol. 2018;44(3):307-315.

17. Jeon MJ, Kim WG, Choi YM, et al. Features predictive of distant metastasis in papillary thyroid microcarcinomas. Thyroid. 2016;26(1):161-168.

18. Ross DS, Litofsky D, Ain KB, et al. Recurrence after treatment of micropapillary thyroid cancer. Thyroid. 2009;19(10):1043-1048.

19. Siddiqui S, White MG, Antic T, et al. Clinical and pathologic predictors of lymph node metastasis and recurrence in papillary thyroid microcarcinoma. Thyroid. 2016;26(6):807-815.

20. Davies L, Welch HG. Increasing incidence of thyroid cancer in the United States, 1973-2002. JAMA. 2006;295(18):2164-2167.

21. Park S, Oh CM, Cho H, et al. Association between screening and the thyroid cancer "epidemic" in South Korea: evidence from a nationwide study. BMJ. 2016;355:i5745.

22. Bychkov A, Jung CK, Liu Z, Kakudo K. Noninvasive follicular thyroid neoplasm with papillary-like nuclear features in Asian practice: perspectives for surgical pathology and cytopathology. Endocr Pathol. 2018;2:1-13.
Cancer Management and Research

\section{Publish your work in this journal}

Cancer Management and Research is an international, peer-reviewed open access journal focusing on cancer research and the optimal use of preventative and integrated treatment interventions to achieve improved outcomes, enhanced survival and quality of life for the cancer patient. The manuscript management system is completely online and includes

\section{Dovepress}

a very quick and fair peer-review system, which is all easy to use. Visit $\mathrm{http}: / /$ www.dovepress.com/testimonials.php to read real quotes from published authors. 